\title{
Malformations cardiaques congénitales par la paroxétine
}

Tobias Leniger

Correspondance:

PD Dr Tobias Leniger

Swissmedic

Division ATC I

Hallerstrasse 7

CH-3000 Berne 9

tobias.leniger@swissmedic.ch
L'entreprise GlaxoSmithKline SA a transmis à Swissmedic de nouvelles données issues de deux études jusqu'alors non publiées sur les risques de malformations congénitales chez les nouveaunés de mères traitées par la paroxétine (Deroxat ${ }^{\circledR}$ ) pendant le premier trimestre de grossesse. Ces résultats montrent un risque accru de malformations congénitales cardiaques (surtout anomalies du septum interventriculaire et du septum auriculaire) chez les nouveau-nés de mères ayant pris de la paroxétine pendant les trois premiers mois de grossesse.

D'entente avec Swissmedic, l'information sur le médicament a été modifiée (cf. ci-après) afin d'en améliorer la sécurité d'emploi. Cette exigence vaut aussi bien pour la préparation originale, le Deroxat ${ }^{\circledR}$, que pour toutes celles qui contiennent de la paroxétine et qui sont commercialisées par d'autres laboratoires pharmaceutiques, à savoir: Parexat ${ }^{\circledR}$ (Spirig Pharma SA), Paroxetin-Mepha ${ }^{\circledR}$ (Mepha Pharma SA), Dexantol $^{\circledR}$ (Medika SA), Paronex ${ }^{\circledR}$ (Sandoz Pharmaceuticals SA), Paroxetin HelvePharm ${ }^{\circledR}$ (Helvepharm SA), Paroxetin Sandoz ${ }^{\circledR}$ (Sandoz Pharmaceuticals SA) et Paroxetop ${ }^{\circledR}$ (G. Streuli \& Co. SA).

Dans son communiqué du 4 octobre 2005, Swissmedic indiquait déjà que la société GlaxoSmithKline SA avait envoyé des analyses de résultats d'une étude rétrospective menée par GSK sur la prévalence des malformations congénitales chez les nouveau-nés de mères ayant pris des antidépresseurs pendant le premier trimestre de grossesse. Confirmant la première analyse intermédiaire (cf. communiqué du 4 octobre 2005), la dernière analyse en date, qui porte sur un plus grand nombre de cas (5956 enfants nés de 5791 femmes ayant pris des antidépresseurs pendant le premier trimestre de grossesse), montre un risque accru de malformations congénitales (rapport des cotes [RC] 1,82; $95 \%$ IC: 1,17-2,82), et en particulier de malformations congénitales cardiaques (RC 1,54; $95 \%$ IC: 0,81-2,92) pour la paroxétine en comparaison d'autres antidépresseurs. Neuf des douze malformations congénitales cardiaques observées chez des nouveau-nés de mères traitées par la paroxétine étaient des anomalies du septum interventriculaire. Si l'on compare ces données avec celles de la population totale (c.-à-d. prévalence des malformations congénitales chez tous les nouveau-nés, indépendamment de la prise d'antidépresseurs pendant la grossesse), il s'avère que la prévalence des malformations congénitales, en particulier cardiaques, est supérieure chez les enfants de mères ayant pris de la paroxétine que dans la population totale $(3,8 \%$ versus $3 \%$ et $1,5 \%$ versus $1,0 \%$ ).

Dans une deuxième étude, ce sont les données du registre suédois des naissances qui ont été analysées. Il en est ressorti un risque presque deux fois plus élevé de malformations cardiaques chez les enfants de mères ayant été traitées par la paroxétine par rapport à la population totale. Cette analyse a également mis en évidence que les anomalies concernaient principalement le septum interventriculaire et le septum auriculaire. Ce risque accru n'a en revanche pas pu être démontré lorsque les femmes avaient pris d'autres inhibiteurs sélectifs de la recapture de la sérotonine (IRSS) que la paroxétine pendant le premier trimestre de la grossesse.

Il convient en outre de souligner que les malformations cardiaques observées sont courantes et qu'elles peuvent aussi bien être asymptomatiques/ne nécessiter aucun traitement qu'être symptomatiques/nécessiter un traitement. Au vu du risque actuel de malformations congénitales, il n'est pas indiqué d'envisager l'interruption de grossesse chez les femmes enceintes traitées par la paroxétine. Swissmedic et les entreprises concernées ont convenu de modifications de l'information sur le médicament (cf. ci-après), qui contient désormais les nouvelles recommandations suivantes sur le risque de malformations associé à l'utilisation de la paroxétine mis en évidence à ce jour:

- De la paroxétine ne devrait pas être nouvellement prescrite chez les femmes qui envisagent prochainement de devenir enceintes ou celles qui sont déjà enceintes.

- Le médecin devra envisager l'option de traitements alternatifs chez les femmes enceintes et déjà traitées avec la paroxétine et ne continuera à prescrire la paroxétine que si cela est absolument nécessaire. Une interruption brutale du traitement doit également être évitée lors de la grossesse.

Ces informations et recommandations ont également été transmises aux médecins sous forme d'une «Dear Doctor Letter» et ont été mises en ligne sur le site web de Swissmedic (sous «Surveil- 
lance du marché» $\rightarrow$ «Médicaments à usage humain» $\rightarrow$ «Informations importantes»).

Swissmedic recevra de la société GlaxoSmithKline SA un rapport final sur l'étude qu'elle a réalisée et décidera sur la base d'une analyse minutieuse notamment de ces données si d'autres mesures s'imposent.

Enfin, pour compléter autant que possible les données disponibles, il est essentiel pour Swissmedic que les effets indésirables et les grossesses de femmes traitées par la paroxétine et d'autres antidépresseurs soient annoncés le plus systématiquement possible. Nous prions donc en particulier les personnes exerçant une profession médicale de signaler tout effet indésirable en remplissant le formulaire d'annonce jaune prévu à cet effet et en l'envoyant à l'un des centres régionaux de pharmacovigilance. Ce formulaire ainsi que les adresses des centres sont disponibles sur le site Internet de Swissmedic (www. swissmedic.ch, sous les rubriques: «Surveillance du marché», «Pharmacovigilance», «Répertoire général Pharmacovigilance», «Formulaire d'annonce»), en annexe au Compendium suisse des médicaments ou sur simple demande auprès de Swissmedic (tél. 03132202 23).

\section{Modification de l'information professionnelle des préparations à base de paroxétine}

\section{Grossesse/Allaitement}

Des études chez l'animal n'ont pas apporté de preuve directe d'un effet tératogène de la substance.

Des études épidémiologiques récentes sur l'administration d'antidépresseurs pendant le premier trimestre de grossesse rapportent un risque plus élevé de malformations congénitales, en particulier cardiovasculaires (par exemple, anomalies du septum interventriculaire et du septum auriculaire), associé à l'utilisation de la paroxétine. Les données suggèrent que le risque d'avoir un nouveau-né présentant une malformation cardiovasculaire à la suite d'une exposition maternelle à la paroxétine est d'environ 1/50, par rapport à une fréquence attendue pour de telles malformations d'environ 1/100 enfants dans la population générale (cf. «Propriétés/Effets»).

Deroxat ne devrait pas être nouvellement prescrit chez les femmes qui envisagent prochainement de devenir enceintes ou celles qui sont déjà enceintes. Le médecin devra envisager l'option de traitements alternatifs chez les femmes enceintes et déjà traitées avec la paroxétine et ne continuera à prescrire la paroxétine que si cela est absolument nécessaire. Si une décision est prise en faveur de l'arrêt du traitement par la paroxétine chez une femme enceinte, le médecin devra consulter les paragraphes «Posologie/Mode d'emploi - Symptômes de sevrage observés à l'arrêt du traitement par la paroxétine» et «Mises en garde et précautions Symptômes de sevrage à l'arrêt du traitement par paroxétine». Une interruption brutale du traitement doit également être évitée lors de la grossesse.

\section{Propriétés/Effets}

Dans une étude épidémiologique rétrospective récente des Etats-Unis, réalisée chez 5956 nourrissons dont les mères avaient reçu pendant le premier trimestre de grossesse la paroxétine ou un autre antidépresseur, un risque plus élevé de malformations congénitales majeures de tous types a été mis en évidence pour la paroxétine par rapport aux autres antidépresseurs (odds ratio 1,8; intervalle de confiance à $95 \% 1,2-2,8)$. De plus, un risque plus élevé de malformations cardiovasculaires a été déterminé pour la paroxétine par rapport aux autres antidépresseurs (odds ratio 1,5; intervalle de confiance à $95 \% 0,8-2,9)$. Ces données ne tiennent pas compte des femmes qui avaient reçu en plus des antidépresseurs des médicaments tératogènes. Les anomalies du septum interventriculaire constituaient la plus grande partie des malformations cardiovasculaires.

La prévalence des malformations congénitales de tous types et celle des malformations cardiovasculaires chez ces nourrissons étaient de $4 \%$ resp. 1,5\% dans le cas de la paroxétine et de $2 \%$ resp. $1 \%$ pour les autres antidépresseurs. Dans la population totale, ces fréquences s'élèvent à 3\% (malformations congénitales de tous types) resp. 1\% (malformations cardiovasculaires) [Centers for Disease Control and Prevention, USA, et Metropolitan Atlanta Birth Congenital Defects Program Data (MACDP)].

Les nourrissons de 6896 femmes ayant été en contact avec des antidépresseurs en début de grossesse (5123 femmes exposées à des ISRS dont 815 femmes exposées à la paroxétine) ont été examinés dans une étude se basant sur les données du registre de déclaration suédois. Un risque plus élevé de malformations cardiovasculaires a été mis en évidence chez les nourrissons ayant été exposés à la paroxétine par rapport au collectif total du registre (odds ratio 1,8; intervalle de confiance à $95 \% 1,1-2,8)$. La fréquence des malformations cardiovasculaires à la suite d'une exposition à la paroxétine en début de grossesse était de $2 \%$ par rapport à $1 \%$ pour le collectif total du registre. Aucune augmentation du risque global de malformations congénitales n'a été mise en évidence chez les nourrissons ayant été exposés à la paroxétine. 